\title{
CLUSTER MAGNETIC FIELDS FROM LARGE-SCALE STRUCTURE AND GALAXY CLUSTER SHOCKS
}

\author{
Mikhail V. Medvedev, ${ }^{1}$ Luis O. Silva, ${ }^{2}$ and Marc Kamionkowski ${ }^{3}$ \\ Received 2006 February 5; accepted 2006 March 23; published 2006 April 11
}

\begin{abstract}
The origin of the microgauss magnetic fields in galaxy clusters is one of the outstanding problems of modern cosmology. We have performed three-dimensional particle-in-cell simulations of the nonrelativistic Weibel instability in an electron-proton plasma, in conditions typical of cosmological shocks. These simulations indicate that cluster fields could have been produced by shocks propagating through the intergalactic medium during the formation of large-scale structure or by shocks within the cluster. The strengths of the shock-generated fields range from a few nanogauss in the intercluster medium to a fraction of a microgauss inside galaxy clusters. Further amplification of these fields by sheared turbulent motions and gas accretion in clusters may be expected. Thus, cluster fields may be explained without resorting to the amplification of a primordial field.
\end{abstract}

Subject headings: galaxies: clusters: general — large-scale structure of universe — magnetic fields plasmas — shock waves — turbulence

The origin of the magnetic fields of 0.1 to a few microgauss strengths observed in galaxy clusters (Vikhlinin et al. 2001; Taylor et al. 2002; Vogt \& Enßlin 2003) poses one of the most intriguing problems in modern cosmology. The most common explanation invokes the amplification of seed or primordial fields by hydrodynamic turbulence that have been excited during the processes of large-scale structure (LSS) formation. Although there are viable astrophysical mechanisms that can generate seed fields with $B \sim 10^{-16} \mathrm{G}$ or weaker (Harrison 1970; Sicotte 1997; Biermann 1950; Gnedin et al. 2000), recent cosmological simulations (Brüggen et al. 2005) show that structure formation can amplify the field between $z=50$ and the present by a factor of 7000 in cluster centers and by a factor of 10-300 within filaments. Hence, in order to explain the observed intergalactic field, one needs seed fields as strong as $B \gtrsim 10^{-10} \mathrm{G}$. The field amplification by dynamo action (Kulsrud et al. 1997; Malyshkin \& Kulsrud 2002) and/or the small-scale plasma instabilities (Schekochihin et al. 2005) are often considered in this context, but the efficiencies of these processes have never been addressed in realistic cosmological simulations. Scenarios with galactic winds and quasar-driven outflows (Miranda et al. 1998; Furlanetto \& Loeb 2001) can provide fields of such strength, but they are rather localized. It is thus not clear whether they can explain entirely the origin of the intergalactic fields in galaxy clusters.

Here we show that magnetic fields can be produced by collisionless shocks in galaxy clusters and in the intercluster medium (ICM) during LSS formation. Cosmological $N$-body and hydrodynamic simulations of LSS formation (Miniati et al. 2000; Ryu et al. 2003) have shown that shocks with Mach numbers up to $M \sim 100$ are ubiquitous on scales of a few megaparsecs to a few tens of megaparsecs. Theoretical analysis of nonmagnetized collisionless shocks indicates that they can generate subequipartition fields (Moiseev \& Sagdeev 1963; Medvedev \& Loeb 1999; Schlickeiser \& Shukla 2003). We verify this prediction with state-of-the-art numerical simulations. We present here three-dimensional particle-in-cell (PIC) simulations of the nonrelativistic (with $v=0.1 c$ ) Weibel instability (Weibel 1959; Fried 1959) in an electron-proton (with

\footnotetext{
${ }^{1}$ Department of Physics and Astronomy, University of Kansas, KS 66045.

${ }^{2}$ GoLP/Centro de Fisica de Plasmas, Instituto Superior Técnico, 1049-001 Lisbon, Portugal.

${ }^{3}$ California Institute of Technology, Mail Code 130-33, Pasadena, CA 91125 .
}

$\left.m_{p} / m_{e}=100\right)$ plasma, thus guaranteeing a clear separation of the relevant timescales. These simulations are computationally expensive and represent a significant advance over previous studies, which simulated relativistic shocks $(v \sim c)$ in electronpositron or low-mass ratio electron-ion plasmas $\left(m_{i} / m_{e} \leq 16\right)$ (Silva et al. 2003; Nishikawa et al. 2003; Frederiksen et al. 2004). Note that a recently discussed possibility that cluster shocks may produce the magnetic fields seen in galaxy clusters (Fujita \& Kato 2005) was based on the assumption that the results of relativistic simulations will also apply in the nonrelativistic regime. Our nonrelativistic simulations fully confirm theoretical predictions and indicate that LSS shocks can produce magnetic fields of strengths of tens of nanogauss and a few microgauss in the intergalactic medium (IGM) and ICM, respectively.

The mechanism of the field generation at shocks is rather simple (Medvedev \& Loeb 1999; Moiseev \& Sagdeev 1963). As a shock propagates into an ambient medium, it reflects (or scatters) a fraction of the incoming (in the shock frame) particles back into the upstream region, and these then form counterpropagating streams. Both groups of particles (ICM/IGM and reflected particles) have bulk velocities of order the shock velocity $v_{\text {sh }}$; they can also have some thermal spread. Both protons and electrons form the streams, and so both species participate in the instability. ${ }^{4}$ One can consider each charged particle in these streams as an elementary current. Since like currents attract each other, it is energetically favorable for the elementary currents to merge into larger current filaments. This process is inhibited at scales smaller than the plasma skin depth, $\sim c / \omega_{p}\left(\omega_{p}\right.$ is the plasma frequency), by the strong electrostatic repulsion of like charges. In contrast, on large scales, the currents are quasi-neutral because of Debye shielding in a plasma. Hence, the filaments and associated magnetic fields grow rapidly. The process stops when most of the particles become trapped in the produced fields and can no longer amplify the field. This happens when the particle Larmor radius $\rho_{\mathrm{L}}=v_{\perp B} / \omega_{c}\left(v_{\perp B}\right.$ is the particle velocity component transverse to the local magnetic field, and $\omega_{c}=e B / m c$

\footnotetext{
${ }^{4}$ Unlike the ultrarelativistic case, the growth rate of the electrostatic (Langmuir) instability may be greater than that of the Weibel instability. The electrostatic fields are predominantly longitudinal and scatter the particles in the $z$-direction, not over the pitch angle. The overall anisotropy of the streams and, hence, the dynamics of the Weibel instability are not substantially affected. Therefore, we do not consider the Langmuir instability in this Letter.
} 
is the cyclotron frequency) becomes comparable to (or less than) the characteristic correlation scale $\lambda_{B}$ of the field, $\rho_{\mathrm{L}} / \lambda_{B} \sim 1$. At this time, the particle distribution is effectively isotropized, and so $v_{\text {thermal }} \sim v_{\perp B} \sim v_{\text {sh }}$.

The anisotropy of the particle distribution near a shock can be parameterized as

$$
A=\left(\epsilon_{\|}-\epsilon_{\perp}\right) / \epsilon_{\mathrm{tot}} \simeq\left(M^{2}-1\right) /\left(M^{2}+1\right)
$$

where $\epsilon_{\|} \propto v_{\mathrm{sh}}^{2}$ is the energy of the particle along the shock propagation direction; $\epsilon_{\perp} \propto v_{\text {thermal }}^{2} \simeq c_{s}^{2}$ is the thermal energy in the plane of the shock; $\epsilon_{\text {tot }}=\epsilon_{\|}+\epsilon_{\perp}$ is the total energy; $c_{s}$ is the sound speed upstream; and the Mach number of the shock is $M=v_{\mathrm{sh}} / c_{s}$. For strong shocks, $M \gg 1$, the anisotropy parameter is close to unity, $A \sim 1$. At a shock, the bulk velocities of the electron and proton components are both comparable to the shock velocity. Hence, the protons dominate over the electrons in the overall energy budget, and the magnetic field generated by the electrons is negligible compared with that generated by the protons. The growth rate and the wavenumber of the fastest growing mode (which, in fact, sets the spatial correlation scale of the produced field) are $\gamma_{B}=A \omega_{p, p}\left(v_{\mathrm{sh}} / c\right)$ and $k_{B}=A \omega_{p, p} / c$, where $\omega_{p, p}=\left(4 \pi e^{2} n_{p} / m_{p}\right)^{1 / 2} \approx 1.32 \times$ $10^{3} n_{p}^{1 / 2} \mathrm{~s}^{-1}$ is the proton plasma frequency, and $n_{p}$ and $m_{p}$ are the number density and the mass of the protons, respectively. (We use cgs units throughout, unless stated otherwise.) The kinetic calculation of the growth rate and the instability threshold have been examined elsewhere (Tzoufras et al. 2006). Order-of-magnitude estimates of the magnetic field $e$-folding time and the field correlation length at strong shocks $(M \gg 1)$ are readily obtained as

$$
\begin{aligned}
& \tau_{B} \sim 1 / \gamma_{B} \simeq 2 \times 10^{2} v_{\mathrm{sh}, 7}^{-1} n_{\mathrm{ICM},-4}^{-1 / 2} \mathrm{~s}, \\
& \lambda_{B} \sim 2 \pi / k_{B} \simeq 10^{10} n_{\mathrm{ICM},-4}^{-1 / 2} \mathrm{~cm},
\end{aligned}
$$

for a typical ICM proton density of $n_{\mathrm{ICM}} \sim 10^{-4} \mathrm{~cm}^{-3}$ and a typical shock velocity $v_{\mathrm{sh}} \sim 10^{7} \mathrm{~cm} \mathrm{~s}^{-1}$; as usual, we denote $n_{\mathrm{ICM},-4}=n_{\mathrm{ICM}} /\left(10^{-4} \mathrm{~cm}^{-3}\right)$ and $v_{\mathrm{sh}, 7}=v_{\mathrm{sh}} /\left(10^{7} \mathrm{~cm} \mathrm{~s}^{-1}\right)$. Since it takes $N \sim$ a few $\times 10 e$-foldings to produce strong fields, we can readily estimate the thickness of a region of the field growth as $\Delta \sim N \tau_{B} v_{\text {sh }} \sim N \lambda_{B}$.

The saturation level of the magnetic field is estimated from $\lambda_{B} \sim \rho_{\mathrm{L}}=v_{\mathrm{sh}} / \omega_{c, p}$, where $\omega_{c, p}=e B / m_{p} c \approx 9.58 \times 10^{3} \mathrm{~B} \mathrm{~s}^{-1}$ is the proton-cyclotron frequency. In a multiple-species plasma, however, saturation occurs at equipartition with the lightest species (Wiersma \& Achterberg 2004). To incorporate this, we introduce an efficiency factor $\eta$, which in the electron-proton plasma is of order $m_{e} / m_{p}$. Finally,

$$
\epsilon_{B}=\frac{B^{2} / 8 \pi}{m_{p} n_{p} v_{\mathrm{sh}}^{2} / 2} \simeq \frac{B^{2}}{8 \pi p_{\mathrm{sh}}} \simeq A^{2} \eta \sim 10^{-3}
$$

where $p_{\mathrm{sh}}$ is the gas pressure behind the shock, and the last estimate is for strong shocks, $A \sim 1$.

Although there is no doubt that magnetic fields are generated at shocks through the Weibel instability, it is not clear whether they survive sufficiently far downstream to produce long-standing magnetic fields. The concern arises from the fact that the wavelength of the fastest growing mode in the linear Weibelinstability analysis is very small, $\lambda_{B} \sim 2 \pi c / \omega_{p, p} \simeq 10^{10} \mathrm{~cm}$ for a typical ICM particle density of $n \sim 10^{-4} \mathrm{~cm}^{-3}$. Therefore, it is possible that the extremely short spatial scales-i.e., sharp field gradients — can be rapidly destroyed by dissipation on a plasma timescale of $\tau_{B} \sim 10^{2} \mathrm{~s}$. Should this happen, the fields would occupy only a very narrow region near the shock front and thus would not result in long-lived cluster fields. Recently, it has been shown (Medvedev et al. 2005), both theoretically and using relativistic PIC simulations, that the correlation length of the magnetic field (and hence its gradient scale) grows rapidly with time, thus drastically reducing diffusive (ohmic) dissipation. These results suggest that the magnetic fields produced should survive on cosmological timescales.

In general, it is far from clear that nonrelativistic shocks can generate fields in the way relativistic shocks do. In order to test this, we have performed a set of three-dimensional and two-dimensional PIC simulations (Dawson 1983; Birdsall \& Langdon 1985) using the state-of-the-art, massively parallel, electromagnetic, fully relativistic, three-dimensional PIC code OSIRIS 2.0 (Fonseca et al. 2002). In our PIC simulations, the initial conditions are taken to be two streams of electrons and ions moving with relative bulk velocity $v_{\text {sh }}$, which in our simulations we take to be $0.1 c$. The four "species" of particles (the upstream and downstream electrons and ions) are then each assigned a Maxwell-Boltzmann distribution of velocities about the bulk velocity. Our ions are "light protons," positively charged particles with mass $m_{\text {ions }}=100 m_{e}$, a mass ratio large enough to guarantee that the electron and ion timescales are clearly separated. All of our simulations have volumes of $128^{3}$ cells, although the cell sizes differ.

We first ran four shorter duration test simulations, three of them three-dimensional and one two-dimensional. In the first three-dimensional run, the box size was $\left(25.6 c / \omega_{p, e}\right)^{3}$ (where $\omega_{p, e}$ is the electron plasma frequency), and there were four particles/species/cell. The second simulation differed from the first only in that the box size was $\left(12.8 c / \omega_{p, e}\right)^{3}$, and the third simulation differed from the first only in that it had eight particles/species/cell. These test simulations showed that neither the box size nor the number of particles per cell affects the results within the available computational resources. We also ran a two-dimensional simulation with $1280^{2}$ cells, i.e., with a box of size $\left(256.0 \mathrm{c} / \omega_{p, e}\right)^{2}$, with periodic boundary conditions and nine particles/species/cell in order to examine the dynamics of a similar system in the plane transverse to the bulk motion of the shocked plasma (the configuration is identical to that in the simulations of Medvedev et al. 2005). A comparison between the three-dimensional simulations and the two-dimensional simulations does not show significant differences (e.g., in terms of the saturation level of the magnetic field); it only reveals the limitations of the two-dimensional simulation (the Weibel instability is stronger in two dimensions) and confirms that the transverse dimensions of the three-dimensional simulation box are not strongly affecting the field dynamics on the timescales analyzed here.

We then ran very long three-dimensional simulations of colliding plasma slabs, for four sets of plasma parameters. One of the plasma slabs describes a shocked high Mach number plasma $\left(M=20, v_{\mathrm{th}, e \text { shock }} / c=0.05\right.$, with either $v_{\mathrm{th}, i \text { shock }} / c=$ 0.005 or $v_{\mathrm{th}, i \text { shock }} / c=0.0$ ) with bulk motion along the $x_{1} \mathrm{di}$ rection. The different ion thermal velocities correspond to the two extreme cases of a strongly turbulent shock (Tidman \& Krall 1971), where electrons and ions in the shocked plasma are thermalized by plasma turbulence and a laminar shock (Sagdeev 1966; Forslund \& Freidberg 1971). The second plasma slab describes the IGM/ICM plasma with either cold electrons, $T_{e}=0$, or hot electrons, $T_{e}=T_{i} \simeq 10 \mathrm{keV}$, such that $v_{\mathrm{th}, e \mathrm{IGM}} / c=0.05, v_{\mathrm{th}, i \mathrm{IGM}} / c=0.005$. All four of these simu- 


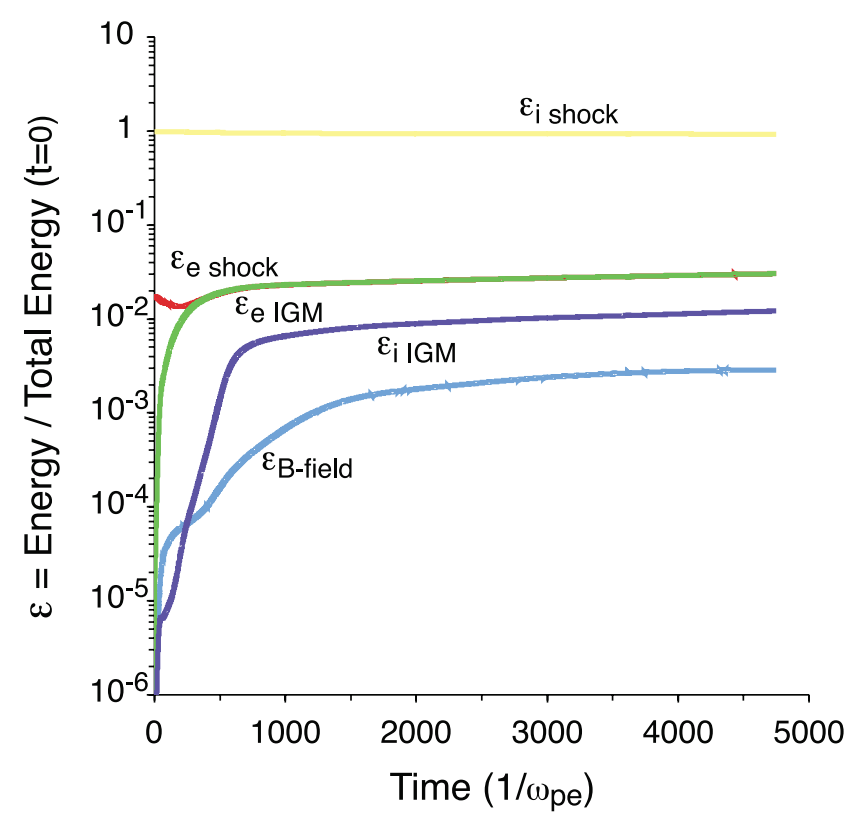

FIG. 1.-The evolution of the magnetic field energy normalized by the total initial kinetic energy, $\epsilon_{B}$, is shown with the light blue line. The energy in the magnetic field is predominantly associated with the field components parallel to the shock plane. For comparison, the similarly normalized energies for the four particle species are also shown.

lations used a box of size $\left(25.6 c / \omega_{p, e}\right)^{3}$. We found that the different physical parameters did not reveal any significant differences in the evolution of $\epsilon_{B}$. Our present choice of simulation parameters is strongly limited by the timescales involved in the mechanisms described here, and it aims to illustrate the key features of the magnetic field generation in conditions relevant for nonrelativistic collisionless shocks.

It is important to note that the structure of a nonrelativistic shock depends on its Mach number, not the shock speed itself. Hence, our simulations with $v_{\mathrm{sh}} / c \sim 0.1$ are rescalable to any thermal velocity of a medium. Thus, for LSS filaments with $T \sim 10 \mathrm{eV}$ and $v_{\mathrm{sh}} \sim 300-500 \mathrm{~km} \mathrm{~s}^{-1}$, our simulations are realistic.

In fact, when examining the temporal evolution of $\epsilon_{B}$ measured in the three-dimensional simulations (Fig. 1), we observe the key role played by the ions, with most of the magnetic field energy generated by the Weibel instability originating in the shocked ions. All the runs revealed $\epsilon_{B} \simeq 10^{-3}$. Note that the Weibel-field growth of the ions saturates at lower relative $\epsilon_{B}$ than for electrons, $\epsilon_{B, i} \sim\left(m_{e} / m_{i}\right)^{1 / 2} \epsilon_{B, e}$, where for species $s=$ $i, e, \epsilon_{B, s}=\left(B^{2} / 8 \pi\right)\left(m_{s} n_{s} v_{\mathrm{sh}}^{2} / 2\right)^{-1}$. A strong thermalization between the electrons in the two slabs is achieved very early in time via the electron Weibel instability, but ion thermalization is not observed in our simulations. Other instabilities with longer timescales (e.g., the ion acoustic instability) will be responsible for this. These instabilities are not observed in our simulations since the simulation box is not large enough.

The timescale for energy transfer between the ions and the magnetic field is the timescale for the Weibel instability of the ions, which is longer than the electron Weibel instability by a factor of $\left(m_{i} / m_{e}\right)^{1 / 2}$, thus making its observation in numerical simulations very time-consuming. The structure of the generated magnetic field depicted in Figure 2 shows the typical configuration of a Weibel-driven field, in three dimensions and in the two-dimensional plane transverse to the bulk motion of

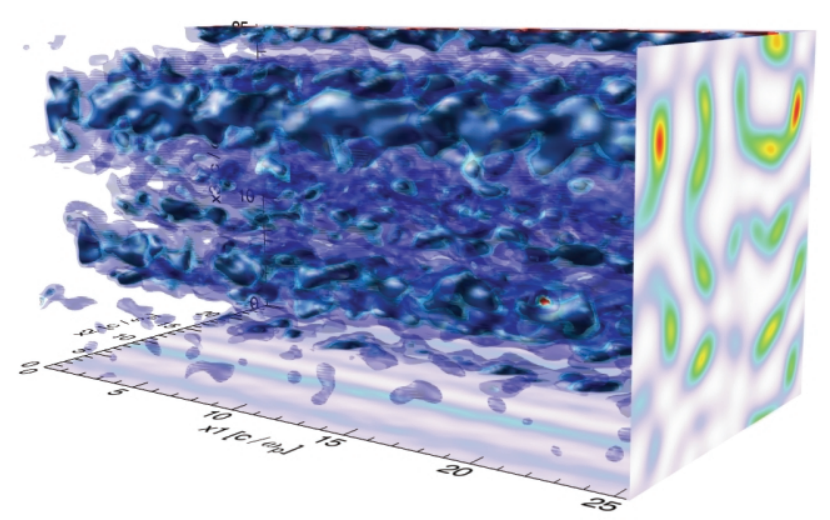

FIG. 2.-Magnetic field energy density at $t=2000 / \omega_{p, e}\left(\omega_{p, e}\right.$ is the electron plasma frequency). The blue isosurfaces correspond to a value of $\epsilon_{B} \simeq 8 \times$ $10^{-3}$. The projection in the $x_{2}-x_{3}$ plane (the shock plane) is the value of $\epsilon_{B}$ averaged along $x_{1}$ (the shock propagation direction), with red color corresponding to a peak value of $\epsilon_{B} \simeq 6 \times 10^{-2}$. The color scale in the projection plane is linear.

the shocked plasma, surrounding the self-generated current filaments, which are already evolving to longer wavelengths.

We have demonstrated that magnetic fields are produced at nonrelativistic collisionless shocks and that their strengths are comparable to that observed in clusters. It is thus natural to explain the observed fields by the Weibel instability. If so, then the magnetization of clusters should begin around the reionization epoch, at redshifts of $z \sim 10-20$, when the gas becomes highly ionized and when particle collisions become rare and inefficient. Our studies reveal that the magnetic field grows to an energy density of roughly a tenth of a percent of the initial kinetic energy density and, hence, constitutes a similar fraction, $\epsilon_{B} \sim 10^{-3}$, of the thermal energy density of the shocked gas. The actual number depends on the complicated nonlinear dynamics of the currents in the downstream region. This value of the equipartition parameter corresponds to a magnetic field strength of order

$$
B \sim 10^{-8} \epsilon_{B,-3}^{1 / 2} v_{\mathrm{sh}, 7} n_{\mathrm{ICM},-4}^{1 / 2} \mathrm{G}
$$

These values correspond to 1 and $10 \mathrm{nG}$ in the ICM (for typical $v_{\text {sh }} \sim 300 \mathrm{~km} \mathrm{~s}^{-1}$ and $n \sim 10^{-6}$ to $10^{-4} \mathrm{~cm}^{-3}$, respectively) and to 0.1 and $1 \mu \mathrm{G}$ inside galaxy clusters (for typical $v_{\mathrm{sh}} \sim 3000$ $\mathrm{km} \mathrm{s}^{-1}$ and $n \sim 10^{-4}$ to $10^{-2} \mathrm{~cm}^{-3}$, respectively). Fields as high as a few microgauss can result from further amplification by turbulent motions in the IGM and by compression as a result of gas infall.

The simulations presented here model a strong shock with Mach number, $M=20$. Can a weak, $M \sim 1$, shock generate fields as well? Three-dimensional simulations of weak shocks are hardly possible at present. However, theoretical analysis of the Weibel instability shows that fields are generated when the shock velocity is larger than the thermal velocity of ICM/IGM particles by a factor of 2 or more, i.e., when $M \gtrsim 2$. The exact number depends on the actual particle distribution at the shock.

LSS shocks can be observed via (1) synchrotron emission by the shock-accelerated electrons in the in situ generated magnetic fields; (2) inverse Compton scattering of cosmic microwave background photons by the shock-accelerated electrons; (3) the Sunyaev-Zeldovich effect on thermal electrons in the shocked medium downstream; and/or (4) an abrupt change in 
the Faraday rotation measure across the shock. The shock front appears to be very thin and will likely be unresolved. Since no sign of proton thermalization is seen by the end of the simulations, $t \sim 500 \omega_{p, p}$, we can put a constraint on the shock thickness,

$$
\Delta_{\mathrm{sh}}>10^{13} n_{\mathrm{ICM},-4}^{-1 / 2} \mathrm{~cm}
$$

Our present analysis does not consider the evolution of the fields on cosmological timescales. Numerical simulations of this type are hardly possible within the next few years. Theoretical considerations suggest that inverse cascade should result in the rapid transfer of magnetic energy from small (shock) to large (cosmological) scales, thus leading to the long-term survival of the fields (Medvedev et al. 2005). An alternative possibility, that other instabilities (Miloslavljevic \& Nakar 2006) present in plasma could destroy the field entirely, is unlikely because the timescales involved are long enough for dynamos driven by turbulence and sheared motions of gas in clusters to further amplify and preserve the shock-generated magnetic fields. A detailed study of these issues is highly important, yet extremely difficult, and should be addressed in the future.

L. O. S. acknowledges the help of Michael Marti in performing the simulations and M. Tzonfres, M. Marti, and Professors Ricardo Fonseca and Warren Mori for discussions. M. K. acknowledges useful discussions with X. Chen, E. Nakar, and M. Milosavljevic. The simulations were performed in the eXpp cluster at IST, Lisbon. The work of M. V. M. has been supported by DoE grant DE-FG02-04ER54790, NASA grant NNG-04GM41G, and the GRF fund. The work of L. O. S. was partially supported by the FCT (Portugal) through grants PDCT/FP/FAT/50190/2003 and POCI/FIS/55905/2004. M. K. was supported by DoE grant DE-FG03-92-ER40701 and NASA grant NNG05GF69G.

\section{REFERENCES}

Biermann, L. 1950, Z. Naturforsch. A, 5, 65

Birdsall, C. K., \& Langdon, A. B. 1985, Plasma Physics via Computer Simulation (New York: McGraw-Hill)

Brüggen, M., Ruszkowski, M., Simionescu, A., Hoeft, M., \& Dalla Vecchia, C. 2005, ApJ, 631, L21

Dawson, J. M. 1983, Rev. Mod. Phys., 55, 403

Fonseca, R. A., et al. 2002, Lecture Notes Comput. Sci., 2331, 342

Forslund, D. W., \& Freidberg, J. P. 1971, Phys. Rev. Lett., 27, 1189

Frederiksen, J. T., Hededal, C. B., Haugbølle, T., \& Nordlund, Å. 2004, ApJ, 608, L13

Fried, B. D. 1959, Phys. Fluids, 2, 337

Fujita, Y., \& Kato, T. N. 2005, MNRAS, 364, 247

Furlanetto, S. R., \& Loeb, A. 2001, ApJ, 556, 619

Gnedin, N. Y., Ferrara, A., \& Zweibel, E. G. 2000, ApJ, 539, 505

Harrison, E. R. 1970, MNRAS, 147, 279

Kulsrud, R. M., Cen, R., Ostriker, J. P., \& Ryu, D. 1997, ApJ, 480, 481

Malyshkin, L., \& Kulsrud, R. M. 2002, ApJ, 571, 619

Medvedev, M. V., \& Loeb, A. 1999, ApJ, 526, 697

Medvedev, M. V., Fiore, M., Fonseca, R. A., Silva, L. O., \& Mori, W. B. 2005, ApJ, 618, L75

Miloslavljevic, M., \& Nakar, E. 2006, ApJ, submitted (astro-ph/0508464)
Miniati, F., Ryu, D., Kang, H., Jones, T. W., Cen, R., \& Ostriker, J. P. 2000, ApJ, 542, 608

Miranda, O. D., Opher, M., \& Opher, R. 1998, MNRAS, 301, 547

Moiseev, S. S., \& Sagdeev, R. Z. 1963, J. Nucl. Energy C, 5, 43

Nishikawa, K.-I., Hardee, P., Richardson, G., Preece, R., Sol, H., \& Fishman, G. J. 2003, ApJ, 595, 555

Ryu, D., Kang, H., Hallman, E., \& Jones, T. W. 2003, ApJ, 593, 599

Sagdeev, R. Z. 1966, Rev. Plasma Phys., 4, 23

Schekochihin, A. A., Cowley, S. C., Kulsrud, R. M., Hammet, G. W., \& Sharma, P. 2005, ApJ, 629, 139

Schlickeiser, R., \& Shukla, P. K. 2003, ApJ, 599, L57

Sicotte, H. 1997, MNRAS, 287, 1

Silva, L. O., Fonseca, R. A., Tonge, J. W., Dawson, J. M., Mori, W. B., \& Medvedev, M. V. 2003, ApJ, 596, L121

Taylor, G. B., Fabian, A. C., \& Allen, S. W. 2002, MNRAS, 334, 769

Tidman, D. A., \& Krall, N. A. 1971, Shock Waves in Collisionless Plasmas (New York: Wiley)

Tzoufras, M., et al. 2006, Phys. Rev. Lett., 96, 105002

Vikhlinin, A., Markevitch, M., \& Murray, S. S. 2001, ApJ, 549, L47

Vogt, C., \& Enßlin, T. A. 2003, A\&A, 412, 373

Weibel, E. S. 1959, Phys. Rev. Lett., 2, 83

Wiersma, J., \& Achterberg, A. 2004, A\&A, 428, 365 